\title{
THE EFFECT OF DIFFERENT POST SPACE SURFACE TREATMENTS ON PUSH-OUT BOND STRENGTH OF FIBER POSTS ADHESIVELY BONDED TO ROOT CANAL DENTIN
}

\author{
Mostafa ElSayed AbouShahba*
}

\begin{abstract}
Aim: The purpose of this study was to analyze the effect of different post-space preparation and irrigation protocol on bond strength of resin cement to root canal dentin.

Methods: Teeth were endodontically treated. After post-space preparation every sample was assigned to one of three groups. In each group different preparation and irrigation protocol was performed as follows: $0.9 \%$ saline solution (Group A), 37\% phosphoric acid etch which was activated with an ultrasonic tip then rinsed with $10 \mathrm{~mL}$ of $0.9 \%$ saline solution (Group B), and manipulated with abrasive diamond tapered stone with round end then rinsed with $10 \mathrm{~mL}$ of $0.9 \%$ saline solution for 10 seconds (Group C). Two parts (coronal and apical) of each sample were analyzed by push out test. Push out bond strength measurements were recorded.
\end{abstract}

Results: For coronal part, group C showed the highest significant values followed by Group B followed by Group A. For apical part, group B showed the highest significant values followed by Group A followed by the Group C. For the total; Group B and C showed highest value compared to group A with insignificant difference between B and C. For comparison between Coronal and Apical; Apical section showed a significant lower value compared to Coronal section for all tested groups at $\mathrm{p} \leq 0.05$.

Conclusions: Phosphoric acid etch activated with an ultrasonic tip then rinsed with saline solution appeared to be the best mode of preparation and irrigation when self-etch bonding is used since the adhesion interface is made by a thick smear layer.

KEYWORDS: Fiber post, Push-out, Post space, Bond strength, Smear layer, Ultrasonic activation.

\section{INTRODUCTION}

Intracanal posts might be necessary in endodontically treated teeth in order to increase retention of final restoration. Fiber posts became more popular nowadays because of their esthetic appearance, higher bond strength to root canal, closer elastic modulus to that of dentin and uniform occlusal stress distribution.

\footnotetext{
* Lecturer of Fixed Prosthodontics, Fixed Prosthodontics Department, Future University in Egypt.
} 
Adhesion of these fiber posts to root dentin is challenging due to difficult handling and delivery of adhesives into the root canal, anatomy of root canal and difficult cleaning of the root canal. Also ideal bond to root canal is related to the quality of the hybrid layer and resin tags produced by the resin cement systems. So, self-etch priming systems are mainly used for bonding of fiber posts, since they modify the smear layer and form a complex hybrid layer. ${ }^{(1)}$

Crowns have one smear layer which is a reflection of the composition of the dentine matrix, on the other hand roots have two smear layers; the primary one contains organic and inorganic materials, infectious deteriorated necrotic dentin and microorganism, and another one done by the drill preparation of the post-space which produces a new (secondary) thick smear layer that is rich in rough debris, sealer and gutta-percha remnants. This secondary smear layer is plasticized by frictional heat obtained from the post drill. So, the effective infiltration, penetration and chemical reaction of adhesive systems used in cementation of fiber post is compromised by the cumulative effect of these two layers. Most fiber post failures are root-resin failure in the form of debonding of the weak point at this interface (resin-dentine). So, during dentine surface treatments, it is essential to completely remove the smear layer in order to obtain bonding between the post and the dentin using resin cement. ${ }^{(2)}$

Also chemical root dentine surface treatment agents are used to increase the micromechanical retention of this cement through removing the smear layer, allowing cement penetration into the dentinal tubules, and decreasing the risk of debonding. ${ }^{(3)}$

Since the effective infiltration of self-etch system in the smear layers of the post space still remains a concern, and the main disadvantage of fiber posts is their low bond strength to root canal walls. The present study assessed the effect of different post space surface treatments, on push-out bond strength of fiber posts adhesively bonded to root canal dentin.

\section{MATERIALS AND METHODS}

\section{Teeth preparation:}

Fifteen sound freshly extracted human central incisors stored in artificial saliva were used in this study. The anatomical crowns of these teeth were decapitated $1 \mathrm{~mm}$ above the cemento-enamel junction.

\section{Root canal treatment}

Root canal treatments were performed for the decapitated teeth with rotary files M-Pro( NiTi-Files Innovative Material and Devics. Inc. China), with the use of glide and copious sodium hydrochloride irrigant. The length of the root canals were determined by hand file size 15 and x-ray. After determining the length, and complete patency of the canal with hand file size 15 , the rotary files were used to complete the mechanical treatment of the canal. The master cone sizes were 30 taper 0.6, before obturation an x-ray was done. Resin sealer was used with the master cone to complete the obturation of the canals.

\section{Post space preparation:}

The sealing materials were removed using a diamond bur under highspeed and with water cooling. The post space of each specimen was prepared with $10 \mathrm{~mm}$ depth $^{(3)}$ and a diameter of $1.25 \mathrm{~mm}$ with a specific drill (FiberKleer 4xPentron Clinical 1717 West Collins Orange USA). All procedures were applied by the same operator, in an effort to standardize all the variables. Following post space preparations, specimens were randomly divided into three groups of 5 teeth each:

\section{Group A:}

The canals were rinsed with $10 \mathrm{~mL}$ of $0.9 \%$ saline solution for 10 seconds.

\section{Group B:}

The canals were filled with $37 \%$ phosphoric acid etch which was activated with an ultrasonic 
tip [E4D tip (Varios, NSK Nakanishi Inc., Kanuma, Japan)], which was mounted on an ultrasonic handpiece (Varios 370, NSK Nakanishi Inc., Kanuma, Japan) with power setting on 7 for 5 seconds then rinsed with $10 \mathrm{~mL}$ of $0.9 \%$ saline solution for 10 seconds. ${ }^{(1)}$

\section{Group C:}

The canals were manipulated with abrasive diamond tapered stone with round end then rinsed with $10 \mathrm{~mL}$ of $0.9 \%$ saline solution for 10 seconds (Active control).

\section{Bonding of fiber posts}

After preparation and irrigation, the canals were dried using paper-points. Pre-silanated Fiberposts (FiberKleer 4x Pentron Clinical 1717 West Collins Orange USA) were used in this study, with diameter of $1.25 \mathrm{~mm}$, the fiberposts were checked to fit in the prepared canals, then removed and cleaned by alcohol before bonding procedure. Dual cure Selfadhesive resin cement (Breeze manufactured by Pentron Clinical 1717 West Collins Orange USA) was applied directly in the canals, then the fiberposts were immediately placed and stabilized in the post spaces. Excess cement was removed after light curing for 2 seconds, for easily removal of excess cement, then complete light curing for 40 seconds to allow complete polymerization of the cement.

\section{Push-out test}

Teeth are embedded in chemical cured acrylic resin and then cross sectioned using [IsoMet 4000 microsaw Buchler USA mounting diamond disk (0.6mm thick)] at speed $2500 \mathrm{rpm}$ and feeding rate 10 $\mathrm{mm} / \mathrm{min}$ under water cooling and $2 \mathrm{~mm}$ thick slices of apical and coronal root portion were prepared for assessment of push out bond strength. Apical and coronal aspects of each sample were photographed and examined using stereomicroscope (Nikon MA100 Japan). Absence of dentin cracks or voids of the filling materials were confirmed.
The post was then loaded with $0.9 \mathrm{~mm}$ diameter stainless steel plunger. The plunger was mounted on an upper part of a universal testing machine (Instron universal testing machine model 3345 England), data were recorded using a computer software (Bluehill 3 version 3.3). The samples were aligned over a support jig in apical and coronal directions to avoid any constriction interference. The tests were conducted at a cross head speed of $0.5 \mathrm{~min}-1$ using a $500 \mathrm{~N}$ load cell. The highest values recorded were taken as the push out bond strength.

The area load was calculated by:

The push out test values in MPa were calculated from force $(\mathrm{N})$ divided by area $\left(\mathrm{mm}^{2}\right)$. [Area= circumference of restoration thickness].

\section{RESULTS}

The results of the push-out bond-strength measurements are presented in Table (1) and Figure (1).

Data showed parametric distribution. Data showed parametric distribution. One-way ANOVA was used to show the difference between tested groups followed by Tukey's (HSD) post hoc test for pairwise comparison. Independent t-test used to compare between tested sections. $(\alpha=0.05)$ (IBM Corp. Released 2015. IBM SPSS Statistics for Windows, Version 23.0. Armonk, NY: IBM Corp.)

1. For coronal part with $p$ value $\leq 0.001$, group c showed the highest significant values $(7.50 \pm 0.79)$ followed by Group B $(5.25 \pm 1.80)$, and the lowest values were for Group A(1.93 \pm 0.46$)$.

2. For apical part with $p=0.001$, group B showed the highest significant values $(2.76 \pm 0.71)$ followed by Group A $(0.56 \pm 0.42)$ followed by the lowest values for Group C $(0.32 \pm 0.25)$.

3. For the total with $p=0.001$, Group B and C showed highest values $(4.00 \pm 1.84$ and $3.91 \pm 3.84)$ compared to group A $(1.24 \pm 0.83)$ with insignificant difference within (B and C). 
4. For comparison between Coronal and Apical parts; apical section showed a significant lower value compared to Coronal section for all tested groups at $\mathrm{p} \leq 0.05$.

TABLE (1) Push-out test for different tested groups.

\begin{tabular}{|l|r|r|r|r|r|r|l|}
\hline \multirow{2}{*}{} & \multicolumn{2}{|c|}{ Group A } & \multicolumn{2}{c|}{ Group B } & \multicolumn{2}{c|}{ Group C } & \multirow{2}{*}{-value } \\
\cline { 2 - 7 } & Mean & SD & Mean & SD & Mean & SD & \\
\hline Coronal & $1.93^{\mathrm{a}}$ & 0.46 & $5.25^{\mathrm{b}}$ & 1.80 & $7.50^{\mathrm{c}}$ & 0.97 & $\leq 0.001^{*}$ \\
\hline Apical & $0.56^{\mathrm{a}}$ & 0.42 & $2.76^{\mathrm{b}}$ & 0.71 & $0.32^{\mathrm{c}}$ & 0.25 & $0.001^{*}$ \\
\hline Total & $1.24^{\mathrm{a}}$ & 0.83 & $4.00^{\mathrm{b}}$ & 1.84 & $3.91^{\mathrm{b}}$ & 3.84 & $0.001^{*}$ \\
\hline
\end{tabular}

Means with the same letter within each row indicates insignificant difference at $p>0.05$

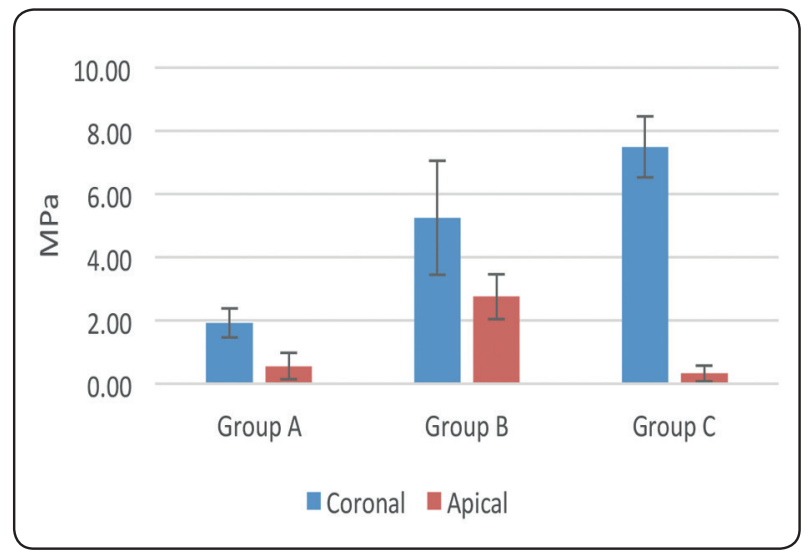

Fig. (1) Bar chart showing the push-out bond strenght.

\section{DISCUSSION}

Application of posts is necessary to provide retention for the core material. Ideally posts should transfer the load to the tooth in order not to cause any root fracture. Fiber post corrosion resistance and elastic modulus enhance the fracture resistance of endodontically restored teeth. ${ }^{(4)}$ Adhesive resin systems were recommended for the cementation of fiber posts in order to distribute stresses over the entire bonded surface and increase retention, but in order to eliminate the problem related to the moist root canal dentin, self-adhesive systems became the choice since they can be applied with wet or dry methods. But many variables may influence the quality of adhesion at the post-cement-adhesivedentininterfaces.Severaldifferentmechanical testing methods have been used to measure the strength of bond between fiber posts and intraradicular dentin, including microtensile bond-strength testing, pullout and push-out tests. The push-out test has been widely used in laboratory research to evaluate the effect of root canal treatment prior to cementing the posts on retention of intracanal posts since it is reliable and reproducible than microtensile test and it is more similar to the clinical condition. ${ }^{(5)}$

When using self-etching/self-priming adhesive systems, removal of smear layer and opening of dental tubules is recommended, because of the thick smear layer produced by drilling during post space preparation which might prevent effective etching. Chemical agents, such as $\mathrm{NaOCl}, \mathrm{H} 2 \mathrm{O} 2$, EDTA, chlorhexidine digluconate, citric acid (10\%, $20 \%$, and 50\%), orthophosphoric acid (H3PO4), and combinations of these, have been previously proposed for the removal of the smear layer. ${ }^{(6)}$

In order to create ideal conditions for optimal adhesion of fiber posts to root canal dentin complete elimination of gutta percha remnants and smear layer from post space is a requirement. The procedure includes drilling in order to remove the filling materials and prepare the post space as well as the use of a cleaning regimen to eliminate the created smear layer. During the drilling sealer and gutta percha residues get plasticized by bur friction heat and inorganic components, but drills achieved an effective post space debridement only when combined with an adequate cleaning regimen.

According to a previous study by Coniglio et al ${ }^{(7)}$, the etching treatment with $35 \%$ phosphoric acid followed by water rinsing was not able to clean dentin optimally; large areas covered by smear layer and sealer/gutta percha remnants were evident on post space walls after etching. So, in the present 
study, the etching treatment was not performed alone but followed by activation with an ultrasonic tip since Kuah et al. ${ }^{\left({ }^{(8)}\right.}$ reported that the combination between irrigants and ultrasonics significantly improved the smear layer removal after endodontic instrumentation.

It appeared in many other studies that ultrasonic cleaning (agitation) mechanically removed the thick plasticized smear layer and subsequently induced a normal smear layer, which is suitable for proper bonding of adhesive system. These studies after evaluating the effectiveness of ultrasonic agitation on smear layer removal from the root canal wall gave some statements as with Mozo et $\mathbf{a l}^{(9)}$ and Srirekha et $\mathbf{a l}^{(\mathbf{1 0 )}}$ who stated that passive ultrasonic agitation with different irrigants to remove root canal smear layer after post space preparation regardless of the type of irrigant, effectively removed smear layer in contrast to the control group and this was in total agreement with the results of this study. Usually manufacturers recommend the pretreatment of dentin with sodium hypochlorite $(\mathrm{NaOCl})$ before post cementation, but it was found in many studies that $\mathrm{NaOCl}$ solution releases active chlorine, which combines with the amine group of proteins to form chloramines, and this can cause strong inhibition of polymerization at the interface of adhesive materials decreasing the bond strength of some adhesive systems. So, based on the results of Seballos et al. ${ }^{(11)}$ it can be concluded that $\mathrm{NaOCl}$ and $\mathrm{CaOCl}$ negatively affected the bond strength between root dentin and fiber posts when cemented with self-adhesive resin cement and should not be used to irrigate the post-space. On the other hand, saline solution promoted the highest bond strength and could be the preferred intracanal solution before fiber post cementation when self-adhesive resin cement, despite its lack of disinfection, based on that $0.9 \%$ saline solution was used as the irrigant in this study for the three tested groups.

Dentin surface prepared by ultrasonic device displayed greater contact surface for bonding process as found in this study with group B when we used phosphoric acid etch and agitated it with ultrasonic tip and revealed a significant increase in the bond strength of fiber post to root canal with this ultrasonic agitation in comparison to groups $\mathrm{A}$ and $\mathrm{C}$ in both the coronal and the apical root segments. A study by Coniglio et al. ${ }^{(7)}$ also highlighted the possibility of reaching an adequate level of cleaning due to the combination of ultrasonic tips and chemicals.

This was in again accordance with what was shown by Plotino et al. ${ }^{(12)}$, who stated that the ultrasonic vibration increased the effectiveness of irrigating solutions in removing debris.

Moreover, Serafino et al. ${ }^{(13)}$ argued that the ultrasonic activation of the etch and the irrigating solution provided effective cleaning even on the apical third of the post space which was in total agreement with the results of the apical bond strength values of group B in this study that showed significantly higher results than both other groups.

It was evident in this study that comparing the coronal parts of the groups demonstrated the increased efficacy of mechanical manipulation over the use of irrigant alone, even more; ultrasonic manipulation of phosphoric acid increased the efficiency of the bond strength above results revealed with regular mechanical manipulation with abrasives. Also ultrasonic agitation of etchant and the action of abrasive diamond tapered stone revealed a positive effect more in the coronal rather than in the apical parts of the post spaces examined.

Furthermore, in the comparison between groups there was a significant difference between the coronal and apical parts because cleaning was more difficult moving apically. In the apical part of the three tested groups, anatomical irregularities impaired access of the (etchant even with ultrasonic agitation/abrasive instrument) to the deepest level and so smear layer manipulation decreased while moving more apical together with different degree of saline (irrigant) penetration along the root walls and this determined a significant difference compared to all coronal parts. Re et al. ${ }^{(14)}$ 
Finally, evaluating all the significant differences found in this study between the three tested groups as well as between the coronal and apical portions within each group can highlight the fact that bond strength between root dentin and fiber post systems is affected by; anatomical features of the teeth (regional number of dentinal tubules that decreases from coronal to apical segments),difficult access to post-space walls, different effect of post space preparations and cleaning instruments and/ or procedures on smear layer that will affect the bonding material penetration, adaptation and polymerization and finally depth of the prepared post-space with lower values generally reported towards the apical segment.

It should be emphasized that generally there was a tendency to statistical significance between groups $\mathrm{B}$ and $\mathrm{C}$ in comparison to group $\mathrm{A}$ that received only irrigation. This statement came in total agreement with the study performed by $\mathbf{R e}$ et $\mathbf{a l}^{(14)}$. Again an agreement was found with Lo Giudice et al ${ }^{(15)}$ who also stated that further confirmation of the effectiveness of ultrasonic activation need to be performed.

Since group B showed a significant difference than the two other groups both coronally and apically; this means that the action of phosphoric acid with ultrasonic activation followed by saline irrigation revealed homogeneous, uniform and effective cleaning and ensured better bond strength results compared to groups $\mathrm{C}$ and $\mathrm{A}$ and is a recommended post space preparation and cleaning method for self-adhesive cementation of fiber posts.

\section{CONCLUSIONS}

Phosphoric acid etch activated with an ultrasonic tip then rinsed with saline solution appeared to be the best mode of preparation and irrigation when selfetch bonding is used since the adhesion interface is made by a thick smear layer

\section{REFERENCES}

1. Kermanshah H, Bolhari B, Sedaghat F, Omrani LR. Effect of Intracanal Post Space Treatments on Push-Out Bond Strength of Fiber Posts to Root Dentin. J Dent. 2017;14(1):55-61.

2. Alaghemand H, Mirzae M, Ahmadi E, Saidi A. Effect of different post-space pretreatments on fiber post bonding to root dentine. Dent Res J (Isfahan). 2013;10(4):545-552.

3. Cintra L, Batistella A, Marson F, Archangelo C, GomesFilho J. Effect of different surface treatments on the pushout bond strength of fiber posts into the root canal. Dent Press Endod. 2015;5(2):33-39.

4. Baldissara P, Zicari F, Valandro LF, Scotti R. Effect of Root Canal Treatments on Quartz Fiber Posts Bonding to Root Dentin. J Endod. 2006;32(10):985-988.

5. Akın A, Toman M, Toksavul S, Sarıkanat M, Firidinog K. The evaluation of displacement resistance of glass FRC posts to root dentine using a thin slice push-out test. Int Endod J. 2009:802-810.

6. Demiryürek EÖ, Külünk Ş, Saraç D, Yüksel G, Bulucu B. Effect of different surface treatments on the push-out bond strength of fiber post to root canal dentin. Oral Surgery, Oral Med Oral Pathol Oral Radiol Endodontology. 2009;108(2).

7. Coniglio, E. Magni, C. Goracci et al. Post space cleaning using a new nickeltitanium endodontic drill combined with different cleaning regimens," Journal of Endodontics, vol. 34 , no. 1, pp. 83-86, 2008

8. Kuah HG, Lui JN, Tseng PS, Chen NN.The effect of EDTA with and without ultrasonics on removal of the smear layer.J Endod. 2009 Mar;35(3):393-6.

9. Mozo S, Llena C, Chieffi N, Forner L, Ferrari M. Effectiveness of passive ultrasonic irrigation in improving elimination of smear layer and opening dentinal tubules. J Clin Exp Dent. 2014;6(1).

10. Srirekha A, Rashmi K, Hegde J, Lekha S, Rupali K, Reshmi G. An in vitro evaluation of passive ultrasonic agitation of different irrigants on smear layer removal after post space preparation: A scanning electron microscopic study. J Indian Prosthodont Soc. 2013;13(3):240-246.

11. Seballos VG, Barreto MS, Abreu R, Machado E, Valandro LF, Kaizer OB. Effect of Post-Space Irrigation with Na$\mathrm{OCl}$ And $\mathrm{CaOCl}$ at Different Concentrations on the Bond Strength of Posts Cemented with a Self-Adhesive Resin Cement. 2018;29:446-451. 
12. G. Plotino, C. H. Pameijer, N. M. Grande and FS. (PDF) Ultrasonics in Endodontics_ A Review of the Literature. Journal of Endodontics. March 2007; 33(2):81-95.

13. Cinzia Serafino, Giuseppe Gallina, Enzo Cumbo, Enzo Cumbo, Francesca Monticelli, Cecilia Goracci MF. Ultrasound Effects after Post Space Preparation_An SEM Study - Journal of Endodontics. June 2006 Volume 32, Issue 6, Pages 549-552
14. Dino Re, Davide Augusti, Gabriele Augusti, Francesca Cerutti, Antonio Cerutti.Cleanliness of dentinal walls following post space preparation using magnification. ENDO (Lond Engl) 2010;4(3):207-214.

15. Giuseppe Lo Giudice, Angelo Lizio, Roberto Lo Giudice, Antonio Centofanti, Giuseppina Rizzo, Michele Runci, Angela Alibrandi and MC. The Effect of Different Cleaning Protocols on Post Space_A SEM Study. International Journal of Dentistry. Volume 2016, Article ID 1907124, 7 pages. 